\title{
Der Stadt-Igel - ein kulturhistorisches Modell für Deutsch als Fremdsprache
}

\author{
Albrecht Classen \\ University of Arizona \\ aclassen@email.arizona.edu \\ https://dx.doi.org/10.12795/futhark.2013.i08.05
}

\begin{abstract}
We have known already for a long time the concept of the "WortIgel" (Word-Hedgehog), and here I introduce the "Stadt-Igel" (CityHedgehog), a pedagogical model with which the teaching of language can be easily combined with the teaching of cultural history and "Landeskunde" (regional and cultural studies). Since the twelfth century cities have become decisive factors in cultural history: most of literature, architecture, art, and music were created there. The "City-Hedgehog" functions in such a way that we choose one city for one German composer/poet. On that basis we can develop countless associations. Moreover, most composers/poets moved from place to place, which allows us, when we pursue a biographical perspective, to develop a whole networtwork of art and city life. In addition, the focus on one artists and poets, to historical events and developments. The City-Hedgehog city makes it possible to create quickly connections to other allows students to perceive better the organic-historical growth of cultural history and to trace that to some extent themselves in working groups.

Keywords: City-Hedgehog, German Cities, German Cultural History, German as a Foreign Language, Music History, Literature History

Abstract: Bekannt ist schon lange der "Wortigel", hier wird nun der "StadtIgel" vorgestellt, ein pädagogisches Modell, bei dem Sprachunterricht mühelos mit Kulturgeschichte und Landeskunde verbunden werden kann. Seit dem zwölften Jahrhundert sind Städte prägend in der Kulturlandschaft geworden; dort entstand die meiste Literatur, Architektur, Kunst und Musik. Der "Stadt-Igel" funktioniert so, dass jeweils eine Stadt gewählt wird zunächst für einen deutschen Komponisten, von dem man ausgehend eine Fülle an Assoziationen entwickeln kann. Außerdem sind natürlich die meisten Komponisten von Ort zu Ort gewandert, was bei einem biografischen Ansatz die Chance bietet, ein ganzes Kultur- bzw. Stadtnetz zu entwickeln.Außerdem erlaubt der Fokus auf eine Stadt, schnell Querverbindungen zu anderen Künstlern und Dichtern, zu historischen Ereignissen und Entwicklungen herzustellen. Der Stadt-Igel ermöglicht den Studenten, das organischehistorische Wachstum der Kulturgeschichte besser wahrzunehmen und dieses in Arbeitsgruppen z.T. selbständig nachzuzeichnen.
\end{abstract}


Schlüsselwörter: Stadt-lgel, deutsche Staedte, deutsche Kulturgeschichte, $\mathrm{DaF}$, Musikgeschichte, Literaturgeschichte, Musik in der Stadt, Literatur in der Stadt

\section{Theoretischer Ansatz}

In diesem Aufsatz geht es darum, ein kulturhistorisch bestimmtes Lehrmodell vorzustellen, das Elemente der Landeskunde mit solchen der Literatur- und Kulturgeschichte verbindet und dies alles so einsetzt, dass eben auch der Sprachunterricht den höchstmöglichen Nutzen davon hat. Wenngleich das Land, der ländliche Raum keineswegs in German Studies vernachlässigt werden soll, repräsentiert doch die Stadt an sich das Zentrum deutscher Kultur, Politik, Wirtschaft und Literatur. Indem wir unsere Studenten primär mit Städten, d.h. deren Geschichte, Strukturen und Relevanz vertraut machen, ermöglichen wir ihnen einen hervorragenden Einstieg, um ihre Sprach- und Kulturkenntnisse zu entwickeln. Bei dem Konzept, das hier vorgestellt und entwickelt werden soll, handelt es sich um den Stadt-Igel, also die Entwicklung eines breitgefächerten Rasters an Assoziationen historischer und zeitgenössischer Orientierung, die eine große Fülle an Herangehensweisen pädagogischer Art ermöglichen und den Sprachunterricht harmonisch in die Bemühungen um die Vermittlung von inhaltlichem Wissen integriert bzw. letzteren Ansatz funktional in den ersteren Aufgabenbereich einfügt.

Es bedarf kaum eines weiten Ausholens, um die allgemeine Selbstverständlichkeit noch einmal hervorzuheben, dass wir im Unterricht Fremdsprache nicht um der Sprache selbst willen vermitteln, auch wenn diese Tätigkeit bzw. dieser Lernprozess aus linguistischen und sprachhistorischen Gründen noch so interessant und wichtig sein mag. Wir lehren auch nicht deswegen eine Sprache, weil dieser intellektuelle Prozess ein irgendwie definierbares ästhetisches Bedürfnis befriedigen würde. Gewiss, weder der eine noch der andere Aspekt dürfte ganz auszuschließen sein, aber er betrifft doch nicht die Hauptabsicht bei DaF bzw. allen anderen Lehrtätigkeiten, die den Fremdsprachenunterricht betreffen. Um es noch einmal ins Gedächtnis zu rufen, wir lehren Sprache, damit unsere Studenten die notwendigen kommunikativen Fähigkeiten erwerben und diese bei der Begegnung mit Sprechern anderer Sprachen einzusetzen vermögen, weiter, damit sie in die Lage versetzt werden, wesentliche oder interessante Texte in einer Fremdsprache zu verstehen, damit sie sich kulturell gesehen ohne zu große Probleme im Ausland bewegen und dort tätig sein können und damit sie Zugang zu

Futhark 8 (2013)

Classen, Der Stadt-Igel, 87-103

ISSN 1886-9300 
wichtigen Informationen gewinnen, die nur in einer Fremdsprache enthalten sind (Hatim 1997). Hinzufügen möchte ich noch, dass mittels DaF den Lernenden der entscheidende Schlüssel in die Hand gegeben wird, kulturhistorische Erforschungen selbsttätig durchzuführen. Dies gilt auch für jeden anderen historisch geprägten Bereich, ob wir an die Medizingeschichte, Musikgeschichte oder Rechtsgeschichte denken, vor allem weil in der deutschen Kulturgeschichte von Anfang an große Beiträge geleistet wurden, die immer wieder die Welt beeinflusst haben (negativ als auch positiv, aber darum geht es hier nicht). DaF muss also stets eine horizontale, zeitgenössische und eine vertikale, historische Perspektive verfolgen.

Aus der Praxis kommend wissen wir alle, dass es viele Jahre dauert, bis unsere Studenten eine Lernstufe erreichen, die als 'near-native' bezeichnet werden könnte. Nur in den seltensten Fällen dürfte es möglich sein, tatsächlich vollkommenen Bilingualismus zu erreichen, es sei denn, es handelte sich um eine Person, die zweisprachig aufgewachsen ist oder sich längere Zeit im Ausland aufgehalten hat. Selbstverständlich können wir als Lehrende nur ein globales Idealziel anpeilen, aber es gibt genauso viele Zwischenstufen dahin, wie es auch Lernende gibt, denn Sprache ist nun einmal ein ureigentlich menschliches Phänomen, komplex, widersprüchlich, identitätsstiftend und kulturschaffend, sperrig und einladend zugleich.

Wesentlich scheint mir daher zu sein, dass wir einen pragmatischen kommunikativen Ansatz verfolgen, denn selbst in Anbetracht der Zielvorgabe, höchste grammatische oder lexikalische Kompetenz zu erreichen, werden wir doch alle eher dahin streben müssen, den Sprachunterricht zumindest so zu organisieren, dass wir unseren Schülern/Studenten eine höchstmögliche kommunikative Kompetenz vermitteln, durch die das erhoffte Wissen oder Verstehen von Daten, Fakten, Ideen oder Vorstellungen erworben werden kann (siehe z.B. die Beiträge in Schramm und Schrader, Hrsgg., 2009; Casper-Hehne, Hrsg., 2009). Solange wir in der Lage sind, die wesentlichen grammatischen und lexikalischen Grundlagen zu schaffen, besteht große Aussicht, darauf aufbauend mit der so zentralen Kulturarbeit zu beginnen, d.h. die entscheidenden sprachlichen Werkzeuge bereitzustellen, den Zugang zu jeglichem relevanten Wissen zu ermöglichen und somit die Studenten zu befähigen, ihre sprachlichen Bemühungen eigenständig fortzusetzen, um weitere Ziele, die jenseits der Sprache an sich liegen, zu erreichen. Diese Beobachtungen wären natürlich nur insoweit gültig, als sie sich auf den Standard DaF-Unterricht beziehen, nicht auf den Bereich der Übersetzungen, wo ganz andere Anforderungen bestehen, die uns aber hier nicht weiter beschäftigen sollen (vgl. dazu Malmkjær und Windle 2011; Pym1992; Hatim und Mason 1995; Venuti 1998; Carbonell 1999). 
Unter 'Kultur' gilt es, ein sehr breites Feld von verschiedensten Aspekten zu verstehen, und am besten scheint mir dies mittlerweile unter der englischen Bezeichnung "German Studies" gefasst zu sein, weil hierbei nicht mehr traditionell ausschließlich auf Literatur, Philosophie oder Religionsgeschichte abgehoben wird, sondern weil der semantische Fächer praktisch jeden Bereich im gesellschaftlichen Leben erfassen kann (vgl. Martinson und Schulz). Natürlich bewahren sich die zentralen Aspekte u.a. von Literatur, Musik und bildenden Künste das Recht darauf, das Hauptaugenmerk auf sich zu lenken, aber wie uns z.B. mittlerweile die Mentalitätsgeschichte, die Gender Studies und Kulturstudien allgemeiner Art vor Augen geführt haben, nähern sich nicht nur diese verschiedenen humanistischen und sozialwissenschaftlichen Bereich im praktischen Kontext aufeinander zu, sondern auch auf der intellektuellen, akademischen Ebene. Dies hat ungemein belebende Wirkungen gerade für $\mathrm{DaF}$, wie ich hier anhand eines, so weit ich sehen kann, neuen Modells, des Stadt-Igels, veranschaulichen möchte. In der Architektur und Kunstgestaltung hat man bereits diese Konzeption praktisch umgesetzt, wie der hölzerne Stadtigel auf dem Forum auf der 4. Internationalen Architektur Biennale in Rotterdam, NAI, 25. Sept. 2009 - 10. Jan. 2010 vor Augen führt (zur Illustration, siehe online: http://www.kaisersrot.com/kaisersrot-02/2009_Stadtigel.html; für weitere Informationen siehe

http://www.alexlehnerer.com/SALE/Stadtigel.html). Schließlich erweist sich die Stadt schlechthin, und dies bereits seit dem hohen Mittelalter, als ein ständig an Bedeutung wachsender Ballungsraum, in dem viele unterschiedliche kulturelle Aspekte und sprachliche Eigenschaften miteinander kondensieren und eine neue Identität bilden (Beiträge in Garber; Noller).

\section{Vom Wort-Igel zum Stadt-Igel}

Das Prinzip der Assoziation beim Erwerb einer Fremdsprache ist ein schon lange bekanntes Modell, das hier nicht breit noch einmal dargelegt werden muss (vgl. z.B. die Beiträge zu Ruiz de Zarobe und Rosa María Jiménez Catalán 2009). Während man sich aber bisher weitgehend mit Wortfeldern beschäftigt hat (Katsaounis), soll es mir hier um ein viel größeres Konzept gehen, das sich produktiv im Sprach-, Kultur- und Literaturunterricht einsetzen lässt. Beim Wort-Igel gehen wir von einem Grundwort aus und suchen dann im Rundumblick nach vergleichbaren, assoziierbaren Worten oder solchen, die etymologisch damit verwandt sind. Die Auswahl mag dann zwar sehr beliebig sein bis hin zur figura

Futhark 8 (2013)

Classen, Der Stadt-Igel, 87-103

ISSN 1886-9300 
etymologica bzw. Volksetymologie (Paretymologie), aber es entstehen dabei wichtige lexikalische Eselsbrücken, die in vielerlei Hinsicht produktiv sein können, insoweit als schnell eine Menge an neuen Wortfeldern erarbeitet werden kann. Ähnlich gestaltet, dann aber doch wesentlich strukturierter, erweist sich der Stadt-Igel. Ursprünglich hatte ich vor der Aufgabe gestanden, einer speziell motivierten Lernergruppe einen besonderen Aspekt der deutschen Kultur- und Literaturgeschichte nahezubringen. Es handelte sich dabei um südkoreanische Musikstudenten, deren Interesse an Deutschland ganz nahe liegt. Der Stadt-Igel besitzt nun genügend an pädagogischem Potential, um für eine unbegrenzte Zahl von Deutschlernenden einsetzbar zu sein. Dies liegt am zentralen Untersuchungsobjekt, die Stadt, die heuristisch gesehen die fundamentale Basis abgibt, um deutsche Geschichte, Kultur, Wirtschaft, Religion, Sport und Politik zu vermitteln.

Wenngleich bis heute touristische Ziele in Deutschland oftmals auf Burgen oder Schlösser bezogen sind, einzelne Kathedralen oder Kirchen herausgegriffen werden oder man sich auf landschaftliche Schwerpunkte stützt (Alpen, Schwarzwald, Ostsee, Lüneburger Heide, der Rhein etc.), können wir weitgehend davon ausgehen, dass letztlich alle unsere Studenten bei ihrem Deutschland-Aufenthalt - die Schweiz und Österreich sollen nachfolgend natürlich immer automatisch mitgedacht werden eine Stadt auswählen und dort die meiste Zeit verbringen. Wie man es auch drehen und wenden mag, die Stadt selbst ist mindestens seit dem Spätmittelalter zu der entscheidenden Zentrale allen Lebens aufgestiegen und hat seitdem sowohl das Kloster als auch den Hof, sowohl die ländliche Gemeinde als auch die adlige Burg überrundet (Lilley 2009; Classen, Hg., 2009). Die (Groß-)Stadt erweist sich immer und überall als der Ort, wo die wesentliche Bildung erworben werden kann, wo die wichtigsten Kulturaufführungen (Theater, Konzert, Ballet und Oper etc.) stattfinden, wo sich die bedeutendsten Bibliotheken befinden, wo die größten Sportereignisse laufen und wo die Politik betrieben wird. Die meisten Länder der Welt sind heute durch ihre Großstädte geprägt, so London, Berlin, Paris, Moskau, Washington DC, Rio da Janeiro, Rom, Kairo, Jerusalem, New Delhi, Tokyo etc. Vor allem die westliche Welt kann spätestens seit der Renaissance als eine urbane Kultur identifiziert werden, selbst wenn weiterhin konkurrierende Institutionen (Kloster, Königshof, Kaserne, die Dorfgemeinde) bestehen. Der Trend hin zum Leben in der Stadt hat sich in der Gegenwart nur noch verstärkt, auch wenn es untergeordnete gegenteilige Bewegungen gibt (zurück aufs Land, in die Natur etc.). Obwohl das Phänomen nicht unbedingt oder explizit angesprochen wird, erweist sich doch gerade DaF in vielerlei Hinsicht als stark orientiert auf die urbane Kultur.

Futhark 8 (2013)

Classen, Der Stadt-Igel, 87-103

ISSN 1886-9300 


\section{Stadt-lgel Musik: Exemplarische Fallbetrachtung}

Im folgenden Abschnitt soll gezeigt werden, wie die pädagogisch ausgerichtete Betrachtung von Städten bzw. ihrer (Kultur)Geschichte dafür benutzt werden kann, um auch Sprachvermittlung zu realisieren. Musik bedarf des Komponisten, des Vortragenden (Praktizierenden) und des Zuhörers. Sehen wir hier von Ausnahmen ab, besonders diejenigen, die das frühe und hohe Mittelalter betreffen, als die Stadtentwicklung noch recht gering gewesen war und die fürstlichen Höfe das dominierende Gewicht besaßen, können wir doch spätestens vom vierzehnten oder fünfzehnten Jahrhundert ausgehend die deutliche Konzentration des Musiklebens auf den städtischen Bereich konstatieren-auch die Höfe befanden sich in den Städten, z.B. Innsbruck, München, Stuttgart etc. Walther von der Vogelweide, oftmals als der 'Sänger des Reiches' apostrophiert (gest. ca. 1220/30) verbrachte zumindest seinen Lebensabend in Würzburg, wo er auch begraben wurde, wenn wir verschiedenen späteren Aussagen (Michael de Leone) trauen dürfen. Allerdings scheint er den größten Teil seines Lebens wie alle anderen weltlichen Sänger seiner Zeit von Hof zu Hof wandernd verbracht zu haben und war noch nicht in einer Stadt ansässig, weil es dort noch nicht das notwendige Auskommen für solche Künstler gab. ${ }^{1}$ Dies war erst etwa 80 Jahre später dem Dichter Konrad von Würzburg (gest. 1287) vorgesehen, dem es gelang, sich fest in Basel niederzulassen und dort viele seiner Werke zu schaffen. Wenn im Literaturunterricht diese zwei Personen behandelt werden, ergibt sich gut die Möglichkeit, vom Mittelalter in die Gegenwart zu springen, Landeskunde zu betreiben und anhand der Stadtbeschreibung die sprachliche Fähigkeiten der Studenten zu stärken, ohne die historische Perspektive aus den Augen zu verlieren.

Ca. 150 Jahre später begegnen wir dem berühmten adligen Dichter Oswald von Wolkenstein (1376/77-1445), dessen Lieder heute als die bedeutendsten musikalischen Werke seiner Zeit jedenfalls im deutschsprachigen Raum angesehen werden. Bemerkenswerterweise kritisierte er häufiger und sehr stark das materialistische Leben in der Stadt, zugleich aber entdecken wir inn häufig in Städten, wo er zu seiner eigenen Erleichterung an der großen Politik im Dienst Kaiser Sigismunds teilnehmen durfte, was seiner eigenen Existenz etwas Glanz vermittelte, während das Leben auf der sehr entfernten Heimatburg Seis am Schlern

1 In Anbetracht der Tatsache, dass ich hier primär pädagogische Aspekte untersuche, unterlasse ich ausführliches Bibliografieren und berücksichtige auch biografisch-historische Aspekte nur ganz flüchtig.

Futhark 8 (2013)

Classen, Der Stadt-Igel, 87-103

ISSN 1886-9300 
nördlich von Bozen in Südtirol oftmals als einsam und bedrückend beschrieben wird.

Wenngleich der gesamte Alpenraum sich in der Gegenwart größter Beliebtheit unter deutschen Touristen, Wanderern u.a. erfreut, scheinen aber jene Regionen um Bozen, Brixen, Meran, dazu Innsbruck oder Hall im außerdeutschen Raum sehr wenig bekannt zu sein. Der Fokus auf Oswald ermöglicht also, die urbane Welt z.B. des heutigen nördlichen Italiens in den Blick zu nehmen und mit den Studenten zu erkunden, wie es zu dem dortigen Wohlstand mit nachfolgender Kunstentwicklung gekommen sein mag, wieso dieser Dichter und Sänger so bedeutend gewesen ist und wie seine Lieder in jener Welt verankert sind. Nebenbei gesagt ergibt sich hierbei die Chance, die historische Entwicklung Südtirols zu behandeln und dem Deutschstudierenden Einblick in die heutige Situation im südlichen Alpenraum vor Augen zu führen, die zu interessanten Diskussionen über Zweisprachigkeit und die Beziehungen zwischen Nord und Süd in Europa einlädt.

$\mathrm{Ab}$ dem 15. Jahrhundert stoßen wir auf immer mehr Komponisten und Liederdichter, von Musikanten ganz zu schweigen, die sich ganz auf die Stadt konzentrierten, dort ihre Existenz besaßen, vortrugen und einen wichtigen Beitrag zur öffentlichen Unterhaltung leisteten. Auf der einen Seite begegnen wir z.B. Martin Luther (1483-1546), der in Wittenberg seine berühmten Kirchenlieder schuf, auf der anderen Seite dem Nürnberger Arzt und Humanisten Georg Forster (ca. 1510-1568), der kurz nach dem Tod des Reformators eine Reihe berühmter Liederbücher herausgab, mit denen er einen ungemein großen Erfolg erlebte, wie die vielen Nachdrucke bestätigen. Forsters Werk lädt somit ein, die wichtige Metropole Nürnberg aus historischer und kultureller Sicht zu begutachten, auf Hans Sachs (1494-1576), Albrecht Dürer (1471-1528) und Catharina Regina von Greiffenberg (1633-1694) u.a. einzugehen, und von dort dann den großen Sprung hin zur Rolle Nürnbergs unter den Nazis und später bei den Alliierten zu machen, um am Ende wieder zu Forster zurückzukehren und einige seiner beliebten Lieder und Balladen zu betrachten, die bis hin zum berühmten Des Knaben Wunderhorn von 1806 wirkten (Classen 2004).

Insbesondere hinsichtlich Luthers ergibt sich die Möglichkeit, den geografischen Rahmen erheblich auszudehnen und produktive pädagogische Ziele hinsichtlich der deutschen Kulturgeschichte zu realisieren, wobei wir oszillierend von dem Bereich Musik wegtreten hin zur Religion und Politik, um dann erneut auf seine berühmten Kirchenlieder sprechen zu kommen. Blicken wir auf Luthers Lebensweg, entdecken wir eine ganze Reihe von wichtigen städtischen Stationen, die individuell betrachtet werden müssten, weil sie jeweils für sich einen großen Einfluss auf die Entwicklungen des Reformers ausgeübt haben: Geburtsort Eisleben, Reichstagsort Worms, wo es zu Luthers Verurteilung kam, 
Eisenach mit der Wartburg, wohin Luther gerettet worden war und wo er sein berühmtes September-Testament 1522 verfasste, und schließlich Wittenberg, wo er seine größte und bedeutungsvollste Wirkung entfaltete und sein Leben als der hoch angesehene Reformator und protestantische Kirchenbegründer beschloss. Nicht zu vernachlässigen wären außerdem Mansfeld, wo er zur Schule gegangen war, Erfurt, wo er studiert hatte, und Torgau, wo er sich mindestens vierzig mal als Prediger oder nur auf Durchreise aufgehalten hatte (auch Halle ist stolz darauf, dass Luther dort dreimal gepredigt hatte, und dort befindet sich auch eine seiner Todesmasken). Auch an Marburg a. d. L. wäre zu denken, weil dort 1529 das berühmte Religionsgespräch mit Zwingli stattfand (s.u.).

Es liegt nun ganz an dem einzelnen Lehrenden, wie intensiv man sich mit den individuellen Städten auseinander setzen oder welchen Querschnitt man anlegen will, aber jede einzelne bietet sich gut und in vielfältiger Weise dafür an, eine Fülle an verschiedenen Aspekten im einzelnen zu verfolgen: Religionsgeschichte, Sozialgeschichte, Musikgeschichte, Literaturgeschichte etc. Außerdem könnte es sich als sehr ergiebig erweisen, zugleich die Architekturgeschichte einzubeziehen, denn Luthers Wohnorte genießen ja heute außerordentlich hohes Ansehen und haben sich zu wichtigen touristischen Zielen entwickelt (Dithmar 2006). Vor allem in der Neuzeit entstanden überall prächtige Opern- und Theaterhäuser, und zugleich verdienen gerade die Kirchen oder Kathedralen, wo die jeweiligen Lieder oder Kompositionen aufgeführt wurden, große Anerkennung. Die praktische Aufgabe bestände nun darin, nicht bloß die Ereignisse des großen Religionskonflikts anhand von Texten zu studieren, sondern zugleich die ungemein wichtige Entwicklung des von Luther im Wesentlichen eingeführten deutschen Kirchenlieds zu untersuchen (Möller). Eine Reihe von lebendigen und weitreichenden Unterrichtseinheiten lassen sich nun mühelos und variabel entwickeln, insoweit als die Thematik der musikalischen Umsetzung der protestantischen Reformation an erster Stelle von politischen und religiösen Aspekten ergänzt und erweitert werden kann. Einzelne Arbeitsgruppen lassen sich aus diesen ersten Ansätzen entwickeln, die im Verlauf des Semesters jeweils die gleichen Linien verfolgen sollten und sich somit zu 'Experten' werden, die bei jeder neuen Kulturepoche die für sie wichtigen Informationen zu liefern in der Lage sein sollten. Der literarhistorische Beitrag schließe sich dann dem musikwissenschaftlichen ganz harmonisch an, und die Mitglieder der jeweiligen Arbeitsgruppe sehen sich dabei unvermutet in die Rolle des Lehrenden versetzt, insoweit als sie der Klasse/dem Seminar ihr eigenes neues Wissen vermitteln.

Futhark 8 (2013)

Classen, Der Stadt-Igel, 87-103

ISSN 1886-9300 
Veranschaulichen wir dies anhand eines Beispiels, um es so konkret wie möglich zu illustrieren. Die wenigsten Studenten werden z.B. mit Eisleben vertraut sein (und dies auch unter deutschen Studenten!). Indem wir eine virtuelle Reise auf der Landkarte durchführen, vermitteln wir den Lernenden zunächst ein Raumkonzept, womit auch wichtige Städte im Osten deutlicher insBewusstsein rücken. In Eisleben 'angekommen,' besteht nun die Aufgabe für einzelne Gruppen, Informationen über individuelle Aspekte dieser Stadt aus historischer oder moderner Perspektive zu sammeln und sie dann in den Unterricht einzubringen. Das Geburtshaus Luthers kann vorgestellt werden, das die anderen beschreiben sollen. Vom Wort 'Geburtshaus' ausgehend kann man nach denen anderer Komponisten in Regensburg, Köln oder Freiburg suchen. Z.B.: Mein Geburtshaus liegt in _, von wo aus ein berühmter Barockkomponist gekommen ist. Er/sie schuf wichtige Symphonien oder Konzerte und gilt heute als .... Oder: Vom Geburtshaus Luthers aus erreicht man schnell das Ratshaus, in dem der Bürgermeister und das Stadtparlament sitzen. Welche anderen bedeutenden Gebäude gibt es in der Stadt? Welche Bedeutung spielt nun Eisleben für die deutsche Religions- und Musikgeschichte? Oder: Außerhalb von Eisleben liegt der früher noch unabhängige Ort Helfta, wo es im Mittelalter ein berühmtes Kloster gab. Wer waren die bedeutendsten Mitglieder dieses Klosters? Welche Art von Musik hat man in einem mittelalterlichen Kloster betrieben?

Dieser komplexe Ansatz lässt sich nun, mit einigen interessanten Modifikationen, hervorragend anhand der Werkes von Johann Sebastian Bach (1685 - 1750) illustrieren, dessen musikalischen Leistungen in vielerlei Hinsicht als grundlegend für den musikalischen Barock angesehen werden. Er wurde in Eisenach geboren, was eine direkte Verbindung zu Luther herstellt, was die Studenten gut lokalgeschichtlich überprüfen können (Eltern, Geburtshaus, Ausbildung dort, erste Wirkung etc. - hier können also sofort die gleichen Satzstrukturen und Wortfelder eingesetzt werden). 1708 bis 1717 finden wir Bach in Weimar, einem wahrlich geschichtsträchtigen Ort in Deutschland. Mehr noch als anderswo beginnt der Stadt-Igel vorzüglich zu greifen, denn anhand von Bach kann eine ganze, breit gefächerte Unterrichtseinheit entwickelt werden jeweils mit Schwerpunkt auf Weimar. Wo und von wem war Bach angestellt, und wie hat die Stadtbevölkerung, abgesehen natürlich vom Hof, wo er tätig war, auf seine Kompositionen reagiert. In welcher Kirche hat er Orgel gespielt? Was ist eine Orgel, und welche anderen Worte lassen sich hier assoziieren? Kennen Sie Konzerte, in denen Orgeln zum Tragen kommen?Welche anderen Musikinstrumente kennen Sie?

Diese horizontale Perspektive lässt sich darauf mühelos durch eine vertikale Sichtweise ergänzen, wenn wir an die 'Weimarer Klassik' (Goethe und Schiller) denken und von dort selbstverständlich den Sprung hin

Futhark 8 (2013)

Classen, Der Stadt-Igel, 87-103

ISSN 1886-9300 
zur 'Weimarer Republik' machen dürfen. Immer wieder ergibt sich hierbei die Möglichkeit bzw. Notwendigkeit, einzelne 'Experten' heranzubilden, die die jeweils wichtigen Puzzlestücke im Verlaufe des Semesters oder der Lehreinheit beitragen. Nicht vergessen werden sollte daher z.B. auch nicht die bittere Tatsache, dass das KZ Buchenwald direkt außerhalb Weimars auf den Bergen liegt und unveränderlich einen Teil der Geschichte Weimars ausmacht. Von der Musikgeschichte gleiten wir dabei ganz unvermutet in die politische Geschichte des 20.Jahrhunderts, aber die musikalische Komponente geht damit nicht verloren. Sogar die jüngsten politischen Entwicklungen in Deutschland sollten hierbei bedacht werden, denn der Freistaat Thüringen hat erst vor kurzem den Antrag gestellt, die Gedenkstätte zu einem Weltkulturerbe zu nominieren (Sept. 2012; http://www.buchenwald.de/index.php?pageid=22\&articleid=399).

Um jedoch nicht völlig die Kleinstadt Weimar übermäßig zu betonen, insoweit sie ja nur auf Grund von Bach einen Ausgangspunkt darstellte, ergibt sich die Notwendigkeit, Bachs Leben weiter zu verfolgen und z.T. sein Leben in Lüneburg und dann vor allem in Leipzig 1723 bis $1750 \mathrm{zu}$ studieren, wo er seine größten Erfolge feierte. Man könnte in dem Zusammenhang sowohl architekturgeschichtlich einen engen Blick auf die Thomaskirche werfen als auch sorgfältige einige von Bachs Kantaten oder Fugen musikwissenschaftlich und aus der Perspektive der Performanz (Gottesdienst) betrachten.

Der Stadt-Igel entwickelt sich von hier aus fast selbständig weiter und kann in alle möglichen Richtungen verfolgt werden. Nehmen wir die Thomaskirche, entdecken wir natürlich leichterdings, welche immense Bedeutung sie im Laufe der Zeit gespielt hat, denken wir daran, dass hier 1409 die Universität gegründet wurde, dass Luther in ihr gepredigt hatte und so die Reformation in Leipzig einführte (1539), dass später Wolfgang Amadeus Mozart 1789, wahrlich in einem europäischen Schicksalsjahr, dort auf der Orgel gespielt hatte, dass Richard Wagner hier 1813 getauft wurde und dass hier schließlich am 9. Oktober 1989, genau wie in der Nikolaikirche, die Protestmärsche gegen das SEDRegime begannen. Die kultur-historischen Assoziationen erweisen sich mithin als unendlich, und wir sehen uns daher auch sofort der Gefahr ausgesetzt, vom Hundertsten zum Tausendsten zu gelangen und jegliche rote Linie zu verlieren. Aber der Rettungsanker besteht ja immer wieder in der zentralen Thematik, der Musikgeschichte, und der inhaltliche Schwerpunkt kann außerdem je nach Vorliebe anders gelegt werden, sei es die Geschichte von Essen, Mode, Gewerbe, Architektur oder Kunst. Musik bietet sich freilich ganz besonders gut an, weil wir hiermit stets öffentliche Kultur greifen, die aufgeführt und eines Publikums bedurfte bzw. von diesem bestellt, gefördert und rezipiert wurde.

Futhark 8 (2013)

Classen, Der Stadt-Igel, 87-103

ISSN 1886-9300 
Je nach individueller Geschmacksrichtung kann sich anschließend die Aufmerksamkeit auf Georg Friedrich Händel (1685-1759) - Halle -, Ludwig van Beethoven (1770-1827) - Bonn -, Amadeus Wolfgang Mozart (1756-1791) - Salzburg - oder Felix Mendelssohn-Bartholdy (18091847) - Berlin et al. - richten, und jedesmal ergeben sich zahllose, höchst produktive and kreative Möglichkeiten, bei der speziellen Stadtbetrachtung weit auszugreifen und über den engen musikhistorischen Zeitpunkt hinaus beliebig viele Querverbindungen herzustellen. Illustrieren wir dies noch einmal speziell anhand MendelssohnBartholdys, der in Hamburg geboren wurde, mit seiner Familie 1811 nach Berlin umzog. 1833 dort die Stelle des Musikdirektors erhielt und 1835 in Leipzig zum Dirigenten des Gewandhausorchester ernannt wurde. Mendelssohn starb zwar in Leipzig, wurde aber in Berlin auf dem Friedhof von Kreuzberg begraben, was viele Anlässe bietet, auch diese wichtige Großstadt kulturhistorisch in den Griff zu nehmen, wenngleich hier natürlich die Gefahr besteht, dass man sich dann gar zu sehr in ein Meer von unendlichen Möglichkeiten begibt und schließlich den kulturhistorischen Zusammenhalt zu verlieren droht. Berlin ist ja wegen seiner zentralen Position schon seit dem 18. Jahrhundert immens bedeutungsvoll gerade für die Kultur- und Kunstgeschichte geworden, vom akademischen und politischen Leben ganz zu schweigen. Immer wieder gilt jedoch, dass die Betonung auf dem Stadt-Igel liegt, der uns die notwendige Rahmenstellung bietet, so dass stets der Weg zurück zum Hauptthema vorgezeichnet ist.

Bleiben wir noch kurz bei Mendelssohn, der natürlich zunächst als Komponist zu berücksichtigen ist, wozu unbedingt auch auf seine Schwester Fanny hinzuweisen wäre, der man wegen ihrer Geschlechtsrolle die gleiche Entfaltungsmöglichkeit als Komponistin wie ihrem Bruder verweigerte. Entscheidend bleibt aber nun, dass Mendelssohn in verschiedenen Städten lebte (einschließlich London), und jede dieser Städte besitzt natürlich eine sehr reiche Geschichte, so dass wir z. B. hinsichtlich Hamburgs auf den dortigen anakreontischen Dichter Friedrich Hagedorn (1708-1754) und viel später auf den Autor Siegfried Lenz (geb. 1926, in Hamburg seit 1948) stoßen, ohne dass damit die Potenzen des Stadt-Igels erschöpft wären. Insoweit als Lenz weiterhin bis heute erstaunlich produktiv tätig ist, könnte man einerseits versuchen, einige seiner jüngsten Publikationen zum Thema zu machen, oder man könnte anhand von ausgewählten Texten den Hintergrund 'Hamburg' und Umland, d.h. die Nordsee und Friesland bzw. dazugehörige Teile wie das 'Alte Land' speziell berücksichtigen, was eine wichtige Fassette ausmacht, wenn wir etwa an die bedeutende Tradition des Orgelbaus u.a. durch Arp Schnitger (1648-1719) denken. Nur nebenbei bietet sich auch die Chance, von Hamburg ausgehend die Geschichte der Hanse aufzugreifen, was eine historisch-geografische Dimension 
eröffnet, und von dort dann kurz auf den Ursprung der deutschen Lufthansa hinzuweisen, womit Vergangenheit und Gegenwart engstens miteinander verknüpft werden. Nur nebenbei bemerkt wurde Johannes Brahms (1833-1897) auch in Hamburg geboren, hatte jedoch in Wien seinen Hauptwohnsitz.Trotzdem wäre es nicht ganz abwegig, hier Verbindungen herzustellen und zu überprüfen, welche Bedeutung Hamburg insgesamt in kulturhistorischer Sicht besessen hatte.

Uns steht immer die Freiheit zur Verfügung, eine mikroskopische oder eine makroskopische Perspektive zu verfolgen, d.h. etwa uns ganz auf den kultur- und zeithistorischen Kontext zu beschränken oder schnell die weiteren Entwicklungen in dieser oder einer anderen Stadt zu berücksichtigen. Eine weitere Alternative besteht darin, den Stadt-Igel entweder ganz auf die musikgeschichtliche Komponente zu limitieren, um mehr präzise Vergleichsmöglichkeiten zur Verfügung zu haben, was etwa bei Leipzig oder Dresden, bei Wien oder München recht einfach in die Tat umzusetzen wäre. Es hängt eben ganz von den Ausgangsbedingungen $a b$, ob wir vor allem mit musikwissenschaftlich interessierten Studenten arbeiten oder solchen, die eine breite kulturhistorische Orientierung verfolgen.

Wie das Beispiel von Hamburg schön vor Augen führt, ergeben sich enorm viele Anknüpfungspunkte und Querverbindungen zwischen wichtigen Musiker-Persönlichkeiten, Literaten und dann auch vielen anderen Figuren des öffentlichen Lebens, die auf der Schiene des StadtIgels unvermutet aber sehr produktiv miteinander verbunden werden können. Es entsteht dadurch ein kulturhistorisches Kaleidoskop, das gerade wegen seiner manchmal doch etwas erratisch wirkenden Charakteristik genau dem zentralen Anliegen entgegen kommt, das wir in DaF bzw. German Studies so intensiv verfolgen, indem wir nämlich uns gerade nicht bloß mit der Sprachvermittlung zufrieden geben, sondern danach streben, den Studenten eine Form des Wissens zu vermitteln, das historische und zeitgenössische Dimensionen umschließt.

Berücksichtigen wir noch das Beispiel von Dresden, wo der bedeutende Hofkomponist Heinrich Schütz (1585-1672) längere Zeit tätig war. Verfolgen wir sein Leben und Werk, bewegen wir uns zunächst auf der historischen Schiene der Renaissance und des frühen Barock und greifen zugleich einen der wichtigsten Musiker seiner Epoche. Er studierte in Marburg a. d. L., was uns eine Reihe von kulturellen Ausgriffen ermöglicht, denn dort war 1527 die erste protestantische Universität der Welt gegründet worden, und dort hatten sich Luther und Zwingli zu ihrem wichtigen Religionsgespräch (1. bis 4. Oktober 1529) getroffen (s.o.). Entscheidend war aber Schütz' spätere Anstellung als Komponist am

Futhark 8 (2013)

Classen, Der Stadt-Igel, 87-103

ISSN 1886-9300 
Dresdner Hof, wo er von 1615 bis zu seinem Tod zutiefst die musikalische Szene, wie wir heute sagen würden, beherrschte. Dresden selbst bietet sich gut an, Stadtgeschichte zu betreiben und eine typische barocke Residenz im urbanen Kontext ins Auge zu fassen (Zwinger). Von hier aus ergeben sich nun eine Reihe von bedeutsamen Assoziationen, die ein Bündel an Impressionen liefern, wenn man eben bereit ist, etwas freier kombinierend wichtige kulturelle Ereignisse, Werke oder Persönlichkeiten zu berücksichtigen.

Angesichts des Zwingers bietet es sich z.B. an, die gegenwärtige Debatte um den jüngst wegen der neuen Elbebrücke verlorenen Status (25. Juni 2009) als Stadt, die von der UNESCO als Welterbe anerkannt worden war, wachzurufen und darüber nachzudenken, welche Bedeutung dieser Status ausmacht und warum sich eine Stadt letztlich nicht mehr darum kümmert, weil logistische Fragen wichtiger geworden sind als historisch-architektonische. Damit aber ist es noch lange nicht getan, denn man kann nun ohne weiteres wieder zurückblicken und mindestens auf zwei bedeutsame und diskussionswürdige Aspekte eingehen.

Zum einen gehört die Tatsache bedacht zu werden, dass Karl May (1872-1912) in Radebeul unweit von Dresden geboren wurde und dann selbst in dieser wichtigen Stadt lebte, ob er nun etwas von Heinrich Schütz wusste oder nicht. So sehr Karl Mays Werk auch zur 'Kultur' mit dem kleinen k gehört, hat er doch mit seinen zahllosen Romanen vor allem über die Kämpfe zwischen Indianern und Weißen in der Neuen Welt einen ungeheuren Einfluss auf eine bis heute sehr umfangreiche Leserschaft vor allem jüngeren Jahrgangs ausgeübt. Meistens gibt es kaum einen Anlass, in DaF, selbst wenn der Fokus auf Literaturgeschichte ruhen mag, sich auch einem Autor wie May zuzuwenden, aber wenn man den Stadt-Igel mit Schwerpunkt auf Dresden verfolgt, erweist es sich geradezu als notwendig, zumindest eine Arbeitsgruppe damit zu beauftragen, sich mit dieser buntschillernden Persönlichkeit auseinanderzusetzen.

Besitzt man etwas mehr Mut, auch brisante Themen anzuschneiden, muss unbedingt anlässslich Dresdens auch das unendliche Leiden der deutschen Zivilbevölkerung gegen Ende des zweiten Weltkriegs berücksichtigt werden, wobei eben die Bombardierung Dresdens vom 13. bis 15. Februar 1945, zu einem Zeitpunkt, als sich eine sehr hohe Zahl von Flüchtlingen dort befand und hilflos den Angriffen ausgesetzt war, nicht außer Acht gelassen werden darf. Gewiss mag der Sprung von Heinrich Schütz und August dem Starken hin zu Karl May und dem Zweiten Weltkrieg etwas gewaltsam wirken, aber akzeptiert man die Relevanz eines Stadt-Igels, fügen sich all diese Teile dann doch recht harmonisch zusammen und sprechen eben unterschiedlichste Interessenlagen an.

Futhark 8 (2013)

Classen, Der Stadt-Igel, 87-103

ISSN 1886-9300 
Wenn z.B. in der Zukunft ein ehemaliger Student der Germanistik aus Geschäftsgründen nach Dresden kommt und Verbindungen mit lokalen Partnern aufnimmt, dann bei den Verhandlungen oder bei sozialen Zusammenkünften sein kulturhistorisches Wissen über Dresden einzubringen vermag, wird er einen entscheidenden Durchbruch bei seinen Gegenüber feststellen, denn er/sie hat ganz unvermutet Gemeinsamkeiten evoziert, die aus der kulturhistorischen Sicht einen wichtigen Vertrauensvorschuss gewährleisten und diesen erheblich ausweiten bzw. stabilisieren. Natürlich kann es dabei auch passieren, dass der deutsche Partner noch nie etwas von Heinrich Schütz gehört hat, aber Karl May und der erbarmungslose Bombenangriff auf Dresden sind tief in das Bewusstsein auch noch der heutigen Bevölkerung eingeprägt, so dass man auf diesem Wege wichtige Kommunikationskanäle zu öffnen vermag.

Will man sich nicht gar zu weit in die Vergangenheit der deutschen Geschichte vorwagen, weil unsere Studenten kaum noch ein an sich zu erwartendes Vorwissen allgemeiner Art darüber besitzen, bietet sich der Stadt-Igel ebenfalls an, nur muss man einfach modernen Perspektiven nachgehen, ohne die spezielle Themenstellung 'Musik' oder Literatur aufgeben zu müssen. Wählen wir zum Abschluss noch einen der bedeutendsten Komponisten des 20. Jahrhunderts, Paul Hindemith (1895 1963), der in Hanau geboren wurde, in Frankfurt a.M. studierte, in Berlin lehrte, in Kairo und Ankara tätig war, 1940 in die USA auswanderte, dort 1946 die amerikanische Staatsbürgerschaft annahm, trotzdem 1953 zurück in die Schweiz kehrte, wo er bis zu seinem Lebensende blieb. Hindemiths atonale Musik erregte den heftigen Zorn der Nazis, was zu vielen Diskussionen im Seminarunterricht führen könnte. Sein Geburtsort Hanau bietet sich hingegen zu Ausflügen in die Welt der Brüder Grimm an, die ebenfalls dort das Licht der Welt erblickt hatten. Hindemiths viele Stationen (Frankfurt, Cairo, Ankara, New Haven, Zürich) laden immer wieder dazu ein, unterschiedlichste kulturhistorische Ausblicke zu machen und viele überraschende Querverweisen zu verfolgen, denn in Frankfurt steht ja das Geburtshaus Goethes, ganz abgesehen von der Tatsache, dass es sich dabei um die finanzielle Zentrale Deutschlands handelt.

Die Kette der vielen wichtigen Komponisten deutscher Zunge lässt sich unablässig weiterspinnen, wobei ich hier nur auf diejenigen verwiesen habe, die zur Kategorie der 'klassischen Musik' gehören. Wie nur zu gut bekannt, hat die deutsche Rock- und Pop-Szene in den letzten Jahrzehnten enorm international an Bedeutung zugenommen, und wenn man so will, kann man den Stadt-Igel genauso auf individuelle RockGruppen und andere Bands ausrichten, um von deren Musik ausgehend Futhark 8 (2013)

Classen, Der Stadt-Igel, 87-103

ISSN 1886-9300 
den breiteren kultur- und literarhistorischen Kontext und Hintergrund auszuleuchten. Gerade weil es sich um einen Stadt-Igel handelt, sind den pädagogischen Möglichkeiten keine Beschränkungen auferlegt, womit auf Grund dieses Lehrmodels viele sprichwörtliche Fliegen mit einer Klappe geschlagen werden können.

Um es noch einmal zu betonen, die Gefahr der Verzettelung, des Sich-Verlierens in einem Wust an Assoziationen ist natürlich stets gegeben, aber selbst im schlimmsten Fall ruht der Fokus immer noch auf deutscher Kultur- oder Literaturgeschichte, die sich auf einmal wegen der vielen verschiedenen Beiträge der studentischen Arbeitsgruppen als ungemein komplex und interessant erweist. Das Konzept des Igels verspricht dabei, unseren Studenten eine ungeahnte Fähigkeit zu vermitteln, allein schon bei der Erwähnung einer Stadt schlagartig auf viele verschiedene Epochen, Persönlichkeiten, Leistungen und Werke einzugehen. Sogar die Landeskunde gewinnt erheblich bei diesem Ansatz, sehen sich ja auf einmal viele unserer Studenten mit einer Zahl wichtiger Städte konfrontiert, von denen sie im traditionellen Unterricht noch gar nichts gehört haben oder noch nichts zu tun hatten. Der Schwerpunkt bei diesem Stadt-Igel ruht nun darauf, dass kleine Gruppen von Lernenden unterschiedliche Aspekte einer Stadt analysieren, weitere Informationen sammeln und somit intensiv mit deutschsprachigem Material arbeiten müssen, das sie nachher selbst an die anderen in der Klasse vermitteln sollen. Der Stadt-Igel bietet sich somit hervorragend dafür an, ein Lerner-orientiertes Lehren in die Praxis umzusetzen. Je nach Sprachstufe können bedeutendere oder alltäglichere Themen verfolgt werden. Die Studenten sind auf jeden Fall dazu aufgerufen, sich auch online oder anderweitig mit komplexem Sprachmaterial auseinanderzusetzen und je nach ihrer Sprachkompetenz die relevante Information herauszufiltern.

Berücksichtigt man z.B. den wohl wichtigsten Komponisten der letzten Jahrzehnte, Karlheinz Stockhausen (1928 - 2007), wandern wir endlich geografisch gesehen in eine Gegend, die bisher noch gar nicht bedacht wurde. Stockhausen wurde in Burg Mödraht bei Kerpen im Kreis Köln geboren, studierte in Köln, war länger in Darmstadt tätig, konzentrierte sich aber die meiste Zeit seines Lebens auf den Kölner Raum. Wir erinnern uns natürlich sofort an Heinrich Böll (1917-1965) oder den Kölner Dom, und von dort kann dann ein ganzes Bündel an Assoziationen abgespult werden. Der Stadt-Igel beweist sich somit als ein Lehrmodell, das auf ganz verschiedenen Ebenen eingesetzt werden kann, die sehr flexibel unterschiedliche Anforderungsstufen besitzen, je nachdem, wie man die Fragestellung formuliert und die konkrete Thematik verfolgt. Als entscheidend könnte sich dann in der Praxis herausstellen, dass die Spracharbeit wesentlich tiefer als bisher in die Landeskunde verankert wird, dass die Lernenden sich dazu aufgefordert sehen, eigenständige Forschungen durchzuführen und dabei zu be-

Futhark 8 (2013)

Classen, Der Stadt-Igel, 87-103

ISSN 1886-9300 
greifen, wie wichtig die eigene Sprachkenntnis ist, um wesentliche Informationen zu sammeln. Zugleich enthüllt sich dabei Landeskunde nicht so sehr als ein geografisch zentriertes Untersuchungsobjekt, sondern als Rahmen, um Literatur- und Kulturgeschichte zu vermitteln, was zugleich auf der Schiene des Deutschunterrichts läuft. Der Stadt-Igel ist letztlich so flexibel, dass das Model für eigentliche jede Lehrstufe eingesetzt werden kann. Auf diese Weise besitzt der Lehrende unendlich viele Möglichkeiten, die Spracharbeit altersgerecht mit Stadt- oder Musikgeschichte zu kombinieren und insgesamt den Lernenden deutlich zu machen, wie wichtig es ist, Deutsch zu lernen, um wesentliche inhaltliche Informationen zu erwerben.

\section{Literaturverzeichnis:}

CARBONELL, Ovidi (1999): Traducción y cultura: de la ideología al texto. Salamanca: Ediciones Colegio de España.

CASPER-HEHNE, Hiltraud (2009): Sprachpraxis der DaF- und Germanistikstudiengänge im europäischen Hochschulraum. Göttingen.

CLASSEN, Albrecht (Hrsg.) (2009): Urban Space in the Middle Ages and the Early Modern Age. Berlin und New York: Walther de Gruyter.

- (2004): Die Rezeption mittelalterlicher Lieddichtung in Des Knaben Wunderhorn. Mediävistische Spurensuche in einem romantischen Meisterwerk. In: Lied und populäre Kultur: Jahrbuch des deutschen Volksliedarchivs 49, S. 81101.

DITHMAR, Reinhard (2006): Auf Luthers Spuren: Ein biografischer Reiseführer. Leipzig: Evangelische Verlagsanstalt.

GARBER, Klaus (Hrsg.) (1998): Stadt und Literatur im deutschen Sprachraum der Frühen Neuzeit. 2 Bde. Tübingen: Max Niemeyer (Frühe Neuzeit, 39).

HATIM, Basil (1997): Communication Across Cultures: Translation Theory and Contrastive Text Linguistics. Exeter: University of Exeter Press.

HATIM, Basil und MASON, Ian (1995): Teoría de la traducción: una aproximación al discurso. Traducción de Salvador Peña. Barcelona: Ariel.

KATSAOUNIS, Nikolaos (2006): "Funkelnde" Wörter: zum Entwurf eines Multimodalen Wortbedeutungsnetzes als gedächtnisstützende und kognitive Lernstrategie im kommunikativen und medial gestützten Unterricht des Deutschen als Fremdsprache. München: Iudicium.

LILLEY, Keith D. (2009): City and Cosmos: The Medieval Woirld in Urban Form. London: Reaktion Books.

MARTINSON, Steve D. und SchULZ, Renate A. (Hrsg.) (2009): Transcultural German Studies: Building Bridges. Bern, Berlin, et al.: Peter Lang (Jahrbuch für Internationale Germanistik. Reihe A. Kongressberichte, 94).

MALMKJ/ER, Kirsten und WINDLE, Kevin (Hrsg.) (2011): The Oxford Handbook of Translation Studies. Oxford und New York: Oxford University Press.

Futhark 8 (2013)

Classen, Der Stadt-Igel, 87-103

ISSN 1886-9300 
MöLLER, Christian (Hrsg.) (2000): Kirchenlied und Gesangbuch: Quellen zu ihrer Geschichte. Ein hymnologisches Arbeitsbuch. Tübingen und Basel: A. Francke.

NOLLER, Peter (2011): Stadt als Raum für Kultur. In: Städte und ihre Eigenlogik: ein Handbuch für Stadtplanung und Stadtentwicklung .Hg. Martina Löw und Georgios Terizakis.Frankfurt a. M. und New York: Campus-Verlag, S. 69-72.

PYM, Anthony (1992): Translation and Text Transfer: an Essay on the Principles of Intercultural Communication Frankfurt a. M., Berlin, et al.: Peter Lang (Publikationen des Fachbereichs Angewandte Sprach- und Kulturwissenschaft der Johannes-Gutenberg-Universität Mainz in Germersheim : Reihe A, Abhandlungen und Sammelbände,16).

RUIZ DE ZAROBE and JIMÉNEZ CATALÁN, Rosa María (Hrsg.) (2009): Content and Language Integrated Learning: Evidence from Research in Europe. Bristol und Buffalo, NY: Multilingual Matters (Second Language Acquisition, 41).

SCHRAMM, Karen und SCHROEDER, Christoph (Hrsg.) (2009): Empirische Zugänge zu Spracherwerb und Sprachförderung in Deutsch als Zweitsprache. Münster, New York et al.: Waxmann (Mehrsprachigkeit, 23).

VENUTI, Lawrence (1998): The Scandals of Translation: Towards an Ethics of Difference. London und New York: Routledge.

Futhark 8 (2013)

Classen, Der Stadt-Igel, 87-103

ISSN 1886-9300 